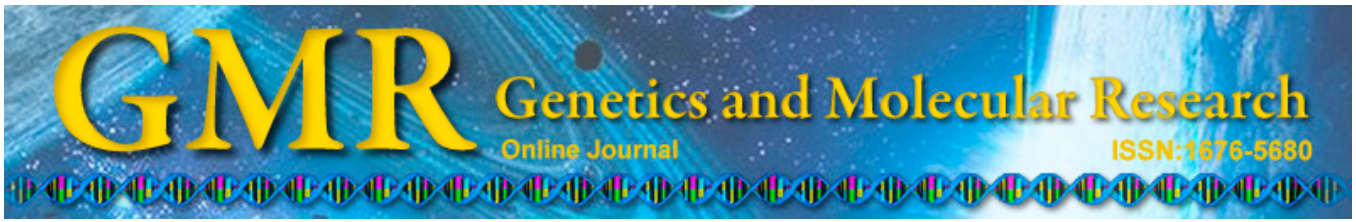

\title{
Body color development and genetic analysis of hybrid transparent crucian carp (Carassius auratus)
}

\author{
W. Xu, G.X. Tong, L.W. Geng and H.F. Jiang \\ Heilongjiang River Fisheries Research Institute, \\ Chinese Academy of Fishery Sciences, Harbin, China \\ Corresponding author: W. Xu \\ E-mail: xwsc23@163.com
}

Genet. Mol. Res. 14 (2): 4399-4407 (2015)

Received June 5, 2014

Accepted November 3, 2014

Published April 30, 2015

DOI http://dx.doi.org/10.4238/2015.April.30.13

\begin{abstract}
The aim of this study was to investigate the genetic mechanism of the transparent trait in transparent crucian carp. We observed body color development in transparent crucian carp larvae and analyzed heredity of color in hybrids produced with red crucian carp, ornamental carp, and red purse carp. The results showed that the body color of the newly hatched larvae matured into the adult pattern at approximately 54 days post-hatching. Two inter-species reciprocal crosses between transparent crucian carp and red crucian carp, and selfcross F1 of transparent crucian carp and self-cross F1 of red marking transparent crucian carp were conducted, and results indicated that the transparent-scaled trait is dominant over the normal-scaled trait. Furthermore, the transparent trait is a quantitative trait. All offspring in the four inter-genera reciprocal crosses of transparent crucian carp with ornamental carp and red purse carp were hybrids of common carp and crucian carp, and had a relatively low survival rate of $10-20 \%$. Moreover, the transparent-scaled trait was observed to be dominant over the normal-scaled trait in the hybrid fish. In conclusion, our results
\end{abstract}


suggest that the genetic mechanism underlying the color of goldfish is complex and requires further investigation.

Key words: Transparent crucian carp; Body color; Development; Hybrid; Genetic analysis

\section{INTRODUCTION}

Goldfish (Carassius auratus) are widespread throughout the world as a famous aquarium fish, known for their bright color, graceful phenotypes, and wide diversity. Previous researchers have demonstrated that goldfish originate from wild crucian carp by studying several aspects of their life history, including their evolutionary history, fish hybridization, embryonic development, karyotype analysis, biochemistry, and molecular biology (Chen, 1928; Fan and Shen, 1990; Komiyama et al., 2009; Wang et al., 2013). Biologists have long been fascinated by the amazing diversity of goldfish color patterns. However, despite much interest, the underlying genetic mechanisms contributing to their rich variety of colors remain largely unknown.

Transparent crucian carp (C. auratus transparent-scaled variant) is a type of goldfish that possesses a number of distinctive characteristics. Specifically, the majority of scales do not have reflective tissue consisting of guanine crystals (guanophore-depleted scales), the head and majority of the body are transparent, it has a single-dorsal fin and a tailed fin, it has a flattened body, the gill filament and viscera are visible during the juvenile stage, and transparency reduces gradually with body growth and thickening of the muscle (Chen, 1928; $\mathrm{Xu}, 2009)$.

It has been shown that body color development in goldfish occurs through differentiation of chromatophores following the depigmentation process after hatching, and the chromatophores that decided body color are black melanophores containing melanin, yellow xanthophores containing pteridine, red erythrophores containing carotenoids, and guanophores (iridophores) containing guanine (Kajishima, 1977; Kawamura et al., 1998; Xu et al., 2005; Leclercq et al., 2010; Nilsson et al., 2013). Transparent crucian carp lose most of their chromatophores from the derma and scales during the depigmentation process in the early stages of development, which renders the scales and tissues transparent.

Previous researchers have no consensus regarding the genetic mechanism of the transparent-scaled character. Berndt (1925) first considered that the transparent-scaled trait was dominant over the normal-scaled trait in goldfish, but shortly after, Chen (1928) demonstrated that the transparent-scaled trait was not completely dominant. Heterozygous fish have transparent and normal scales in mosaic patterns, so that the fish appears mottled in color. In addition, Matsumoto et al. (1960) discovered another type of scale transparency, which was recessive to the normal-scaled trait. Furthermore, Wu and $\mathrm{Fu}$ (1983) suggested that red was dominant over all other colors in goldfish, except for the transparent-scaled character. These observations reflect the complexity of transparent color inheritance in goldfish. In addition, the mutual relationship of transparency and other colors is seldom reported. In view of the body color of transparent crucian carp, we observed body color development in transparent crucian carp larvae and analyzed color heredity in hybrids produced with red crucian carp, ornamental carp, and red purse carp in order to facilitate further research of color heredity in aquarium fish. 


\section{MATERIAL AND METHODS}

\section{Subjects}

Broodstocks were obtained from earthen Ponds on SongPu farm at Heilongjiang Fisheries Research Institute, Heilongjiang, China. White transparent crucian carp (C. auratus. transparent-scaled variant) were from an F5 generation; red crucian carp (C. auratus red variant) were common crucian carp; white ornamental carp (Cyprinus carpio L., Koi) were from an F3 generation resulting from a cross between the Japanese original breed and a cold-resistant strain of ornamental carp; and red purse carp (C. carpio var. wuyuanensis) were a cross-bred cold-resistant strain. In the present study, the majority of scales of the transparent crucian carp (transparent-scaled) did not contain guanophores and were termed "guanophore-depleted". In contrast, "normal-scaled red crucian carp" were termed "guanophore-completed".

\section{Observations of body color genesis in transparent crucian carp}

Newly hatched transparent crucian carp larvae (150 individuals) were selected and maintained in PVC tanks ( $1 \mathrm{~m}$ diameter x $40 \mathrm{~cm}$ high) at room temperature $\left(16^{\circ}-29^{\circ} \mathrm{C}\right)$. Larvae were fed by satiation feeding of Daphnia for 15 days in the early stages of development, and then sufficient artificial feed (Tong Wei feed Co., Guangdong, China) was offered during the later stages of development. During the first 15 days post-hatching (dph), observations were conducted by microscope with an anatomical lens once a day. During 15-45 dph, observations were carried out every other day and then every three days after 45 days. When body color changed extensively, records and photographs were taken.

\section{Color heredity in crosses of white transparent crucian carp and red crucian carp}

Based on the characteristics of the red markings on the surface of transparent crucian carp, we divided individuals into four intermediate phenotypes: white-transparent (no red markings), white-red (red markings on less than half of the body), red-white (red markings on more than half of the body), and red (almost completely red). Based on the number of scales containing guanophores, we divided individuals into five levels $0,1-10,11-20,21-30,>30$ scales containing guanophores. To analyze the genetic mechanism of transparent crucian carp, four crosses were produced. White-transparent crucian carp $(+)$ x red crucian carp ( $\left.\delta^{\Uparrow}\right)$, red crucian carp $(+9)$ x white-transparent crucian carp $\left({ }^{\Uparrow}\right)$, self-cross F1 of white-transparent crucian carp and self-cross F1 of red transparent crucian carp (including white-red and red-white types). In addition, reciprocal crosses of transparent crucian carp with ornamental carp and red purse carp were also conducted.

The induced propagation procedure was conducted according to the method of $\mathrm{Xu}$ et al. (1999), briefly, 8-10 females and males were selected from each cross combination to conduct induced propagation. When the females started to spawn, eggs and sperm from three couples were collected for induced insemination. Larvae (800-1000) from different crosscombinations were stocked in one earthen pond $\left(300 \mathrm{~m}^{2}\right)$ and fed for 5 months. Progeny phenotype analysis was conducted when the weight of individuals reached 30-60 g and body length reached $80-100 \mathrm{~mm}$. Thus, we observed and counted the number of scales containing guanophores in 576 white, 725 white-red, 388 red-white, and 202 red individuals, and calculated the ratio of different guanophore-scale levels in the population. 


\section{RESULTS}

\section{Origin of body color in transparent crucian carp}

To study the origin of body color in transparent crucian carp, we observed and recorded the larvae at different periods. The newly hatched larvae were transparent and appeared light black-grey at $8 \mathrm{dph}$, (Figure 1-1) and large branched melanophores were mainly distributed in the head and middle of the lateral lines (viewed using the anatomical lens). At 9-15 dph, melanophores increased and xanthophores began to gradually appear, and round chromatophore-dots could be seen under the microscope (Figure 1-2 and 1-3). From 16-25 $\mathrm{dph}$, scales began to form, while body color began to differentiate; however, the larvae were reddish and transparent, and the four color phenotypes could not be distinguished at this stage (white-transparent, red-white, white-red, etc.; Figure 1-4). From 26-37 dph, the scales completely formed and body color could be distinguished more clearly (Figure 1-5). At $54 \mathrm{dph}$, the body color of larvae had essentially matured into the adult pattern, which was still transparent, and most scales lacked the reflecting layer (Figure 1-6).

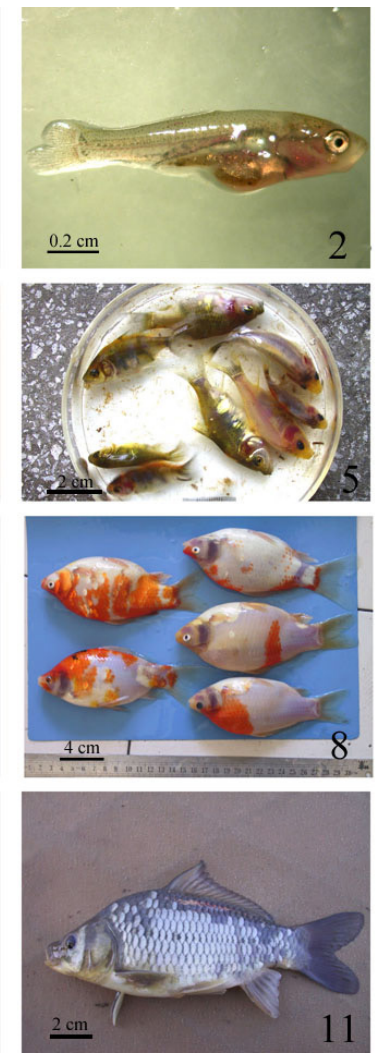
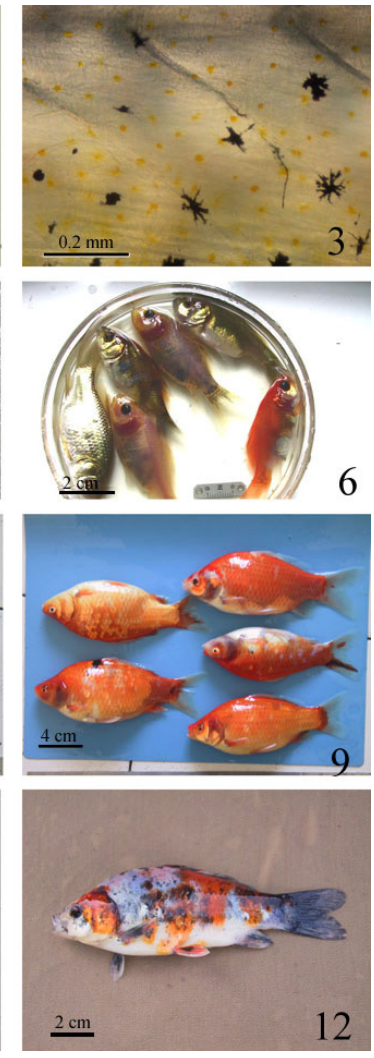

Figure 1. Body color development in hybrid transparent crucian carp. (1) Body color at 8 days post hatching (dph); (2) body color at $14 \mathrm{dph}$; (3) chromatophores at $14 \mathrm{dph}$; (4) body color at 16-25 dph; (5) body color at 26-37 dph; (6) body color at $54 \mathrm{dph}$; (7) white transparent crucian carp; (8) red marking transparent crucian carp; (9) red transparent crucian carp; (10) sepia hybrids; (11) light blue hybrids; (12) red marking hybrids. 


\section{Color heredity in crosses between white transparent crucian carp and red crucian carp}

Reciprocal crosses between white transparent crucian carp and red crucian carp resulted in guanophore-depleted transparent crucian carp offspring. Among the offspring, white color individuals (Figure 1-7) accounted for $10 \%$ of offspring and individuals with red markings (including white-red and red-white fish) and red coloring accounted for $90 \%$ of offspring (Figure 1-8 and 1-9). Self-cross F1 of white transparent crucian carp were all guanophoredepleted transparent crucian carp, and those with red markings or red color (Figure 1-8 and 1-9) accounted for $54.3 \%$ while those with white color accounted for $45.7 \%$ (a segregation ratio approaching $1: 1$ ).

Self-crosses of the red marking transparent crucian carp F1 generation resulted in guanophore-depleted transparent crucian carp and guanophore-completed red crucian carp progeny. The segregation ratio of transparent crucian carp (including the four intermediate phenotypes) and red crucian carp was 74.0 and $26.0 \%$, respectively, approaching $3: 1$. Moreover, among the transparent crucian carp offspring, individuals with red markings or red color accounted for $58.4 \%$ and white individuals accounted for $15.6 \%$ (Table 1).

\begin{tabular}{|c|c|c|c|c|}
\hline \multirow[t]{2}{*}{ Cross combination } & \multirow[t]{2}{*}{ Number } & \multicolumn{2}{|c|}{$\begin{array}{l}\text { Transparent crucian carp } \\
\text { (guanophore-depleted) }\end{array}$} & \multirow{2}{*}{$\begin{array}{c}\begin{array}{c}\text { Red crucian carp } \\
\text { (guanophore-completed) }\end{array} \\
\text { Red }\end{array}$} \\
\hline & & White & Red markings, red & \\
\hline White transparent crucian carp $(+)$ x red crucian carp $\left({ }^{\Uparrow}\right)$ & 654 & 9.9 & 90.1 & 0 \\
\hline Red crucian carp ( $(+) \times$ white-transparent crucian carp $(0)$ & 587 & 12.0 & 88.0 & 0 \\
\hline Self-cross F1 of white transparent crucian carp & 778 & 45.7 & 54.3 & 0 \\
\hline Self-cross F1 of red marking transparent crucian carp & 847 & 15.6 & 58.4 & 26.0 \\
\hline
\end{tabular}

\section{Observations of red markings and guanophore-scale distribution on the body surface of transparent crucian carp}

Through observations and classification of the red markings and guanophore-scale distribution on the body surface of numerous transparent crucian carp, two results were obtained (Figure 2). At the individual level, the body color of transparent crucian carp included the size, number of red markings, and variation in the number of the guanophore-scales that covered the body surface. At the population level, the size, number of red markings, and the number of guanophore-scales was present as a continuously variable trait. Furthermore, we analyzed the ratio of guanophore-scale levels in transparent crucian carp to those in different body color carp to demonstrate that the number of guanophore-scales increases within increasing red markings. Therefore, there is a positive correlation between the number of guanophore-scales and red markings.

\section{Color heredity of the crosses of white transparent crucian carp with ornamental carp and red purse carp}

The four inter-genus reciprocal crosses of transparent crucian carp with ornamental carp and red purse carp showed that all offspring were common carp and crucian carp hybrids, 
which had a relatively low survival rate of $10-20 \%$. Among the hybrid offspring, the guanophore-depleted character was dominant over the guanophore-completed character, which was in accordance with the crosses between white transparent crucian carp and red crucian carp. The proportion of fish with the guanophore-depleted pattern was $80-90 \%$, of which body color mainly appeared sepia (Figure 1-10), light blue (Figure 1-11), and red-white (Figure 1-12), and the proportion of fish with guanophore-completed pattern was $10-20 \%$, of which body color was mainly bluish grey (Table 2).

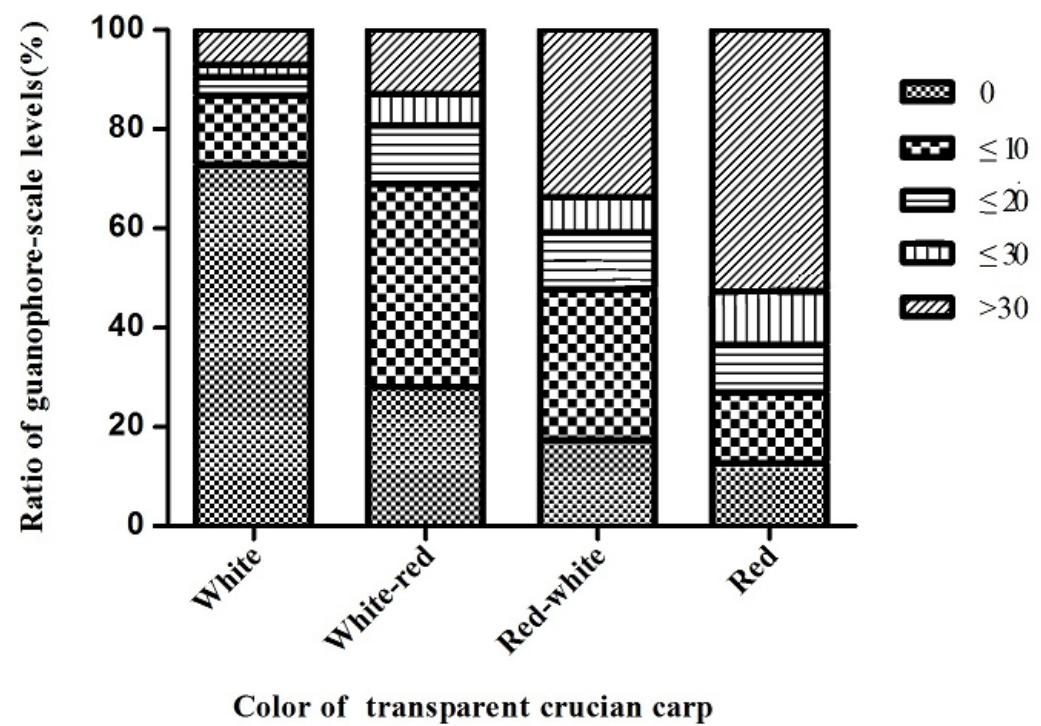

Figure 2. Ratio of guanophore-scale levels in a population of transparent crucian carp with different body colors.

Table 2. Number and ratio of different phenotypes in the offspring of crucian carp crosses (\%).

\begin{tabular}{|c|c|c|c|c|c|c|}
\hline \multirow[t]{2}{*}{ Cross combination } & \multirow[t]{2}{*}{ Number } & \multicolumn{4}{|c|}{ Guanophore-depleted } & \multirow{2}{*}{$\frac{\text { Guanophore-completed }}{\text { Bluish grey }}$} \\
\hline & & Sepia & Light blue & Red markings & Others & \\
\hline White transparent crucian carp $(+)$ x red purse carp $\left({ }^{\lambda}\right)$ & 150 & 56.7 & 30.7 & 2.0 & 1.3 & 9.3 \\
\hline Red purse carp (q) x White transparent crucian carp (ठ) & 97 & 47.4 & 29.9 & 1.0 & 1.0 & 20.6 \\
\hline White transparent crucian carp (O) x ornamental carp $(0)$ & 191 & 51.3 & 31.9 & 1.0 & 3.1 & 12.6 \\
\hline Ornamental carp (q) x White transparent crucian carp $(\widehat{ })$ & 149 & 53.7 & 27.5 & 2.7 & 0.7 & 15.4 \\
\hline
\end{tabular}

\section{DISCUSSION}

Observing ontogenetic development of body color is important to determine the final color pattern in fish. In general, it is impossible to discriminate the phenotype of larvae just after hatching, due to the depigmentation process, which usually takes two to three months after hatching (Goodrich and Hansen, 1931). In our observations of color differentiation in transparent crucian carp larvae, individuals were bluish grey immediately after hatching and took approximately 54 days to complete the demelanization process and mature into the adult pattern. However, researchers have also observed individuals that do not complete the demelanization process, taking 2-3 years for the black pigment (melanin) to fade away and then 
become red (Xu et al., 2005; Wang et al., 2006). These studies indicate that although black is an important component of body color, it can be unstable in goldfish. In addition, there were relatively few offspring with black markings in this study; therefore, we did not specifically classify them. Although there are a variety of colors and types within individuals, we consider that the major changes in color mainly occur in relation to the size and number of red markings, as well as in relation to the number of guanophore-scales covering the body surface.

The results obtained from the reciprocal crosses of white transparent crucian carp and red crucian carp showed that the transparent-scaled trait is dominant over the normal-scaled trait. Self-cross F1 of red marking transparent crucian carp exhibited a segregation ratio of white transparent crucian carp and red crucian carp progeny approaching 3:1, which is consistent with the results obtained by Chen (1928), Kajishima (1977), and Kawamura et al. (1998). Nevertheless, there were $10 \%$ white transparent crucian carp in the reciprocal cross offspring of white transparent crucian carp and red crucian carp, rather than all individuals having red markings, and the offspring of self-cross F1 of white transparent crucian carp had fish with both white and red markings rather than all fish with white markings, which was consistent with the phenomenon found during the inbred process of white transparent crucian carp F5 in our previous study (Xu et al., 2000). These observations are different from those of previous studies conducted on goldfish, which showed that the transparent-scaled trait is not completely dominant over the normal-scaled trait, and does not fit the Mendelian expectations (Chen, 1928; Kajishima, 1977; Kawamura et al., 1998). The incomplete dominance theory of a small number of alleles cannot explain these results. Therefore, we assume that the transparentscaled trait is determined by multiple genetic loci, i.e., polygenic inheritance.

The analysis of the relationships between red markings and guanophore-scales in the population showed that the transparent trait performed as a continuously variable trait. From the above observations, we argue that the genetic mechanism of color in transparent crucian carp is not as clear-cut as previous study has suggested and we demonstrate that the transparent color is a quantitative trait. The variation in phenotypes of transparent crucian carp is typically due to underlying genetic complexity from multiple interacting loci, with allelic effects that are sensitive to the environmental conditions of each individual. However, the differences in our results compared with previous studies may also be caused by different fish species, observation times, and culture conditions (Kawamura et al., 1998; Sugimoto, 2002; Neumeyer, 2003; Leclercq et al., 2010; Nilsson et al., 2013). In the future, it will be interesting to undertake quantitative trait loci mapping to determine which loci affect the transparent trait.

The survival of different fish hybrids is closely related to their genetic structure, and chromosomes. A number of researchers have found that most inter-generic hybrids can fertilize and hatch (Nirchio et al., 2003; Lou and Li, 2006; Hashimoto et al., 2012). This is consistent with our results that demonstrate that fertilization and hatching can be achieved using four inter-generic reciprocal crosses of transparent crucian carp with ornamental carp and red purse carp. The crossed progeny had a similar fertilization rate to other combinations; however, larvae began to experience mortality at $10 \mathrm{dph}$, and a large size variance and relatively low survival rate (about 10-20\%) were observed. The reasons for this may include a genetic defect caused by chromosomal recombination, which did not affect the egg hatching process, but may have induced most individuals to die during growth (Chevassus, 1983; Lou and Li, 2006). There were transparent and bluish grey crucian carp hybrids among the crossed offspring produced by crossing transparent crucian carp with ornamental carp and red purse carp. The dorsal scales lacked reflective tissue in a small fraction of bluish grey carp-crucian carp 
hybrids. As for the transparent hybrids, nearly all individuals with white and red markings died during the growth period in the pond. These results indicate that the genes that control the color of goldfish are complex. Therefore, it is of particular interest to further investigate the molecular mechanism underlying these results.

In conclusion, the present study investigated the body color development process of transparent crucian carp, demonstrating that the transparent trait in the color of goldfish is a quantitative trait and the underlying genetic mechanism is complex.

\section{ACKNOWLEDGMENTS}

Research supported by the Key Research Projects from the Heilongjiang Province Science and Technology Commission, China (\#G96B4-1). We would like to thank the workers of SongPu farm for their aid in breeding and collection of the samples.

\section{REFERENCES}

Berndt W (1925). Verererbungstudien an Goldfischrassen. Zeitshr. f. Indukt. Abst. u. Vererd. 36: 161-349.

Chen SC (1928). Transparency and mottling, a case of Mendelian inheritance in the goldfish, Carassius auratus. Genetics 13: 434-452.

Chevassus B (1983). Hybridization in fish. Aquaculture 33: 245-262.

Fan Z and Shen J (1990). Studies on the evolution of bisexual reproduction in crucian carp (Carassius auratus gibelio Bloch). Aquaculture 84: 235-244.

Goodrich HB and Hansen IB (1931). The postembryonic development of Mendelian characters in the goldfish, Carassius auratus. J. Exp. Zool. Part A 59: 337-358.

Hashimoto DT, Senhorini JA, Foresti F and Porto-Foresti F (2012). Interspecific fish hybrids in Brazil: management of genetic resources for sustainable use. Rev. Aquac. 4: 108-118.

Kajishima T (1977). Genetic and developmental analysis of some new color mutants in the goldfish, Carassius auratus. Genetics 86: 161-174.

Kawamura K, Hosoya K and Matsuda M (1998). Transparent-scaled variant of the Rosy Bitterling, Rhodeus ocellatus ocellatus (Teleostei: Cyprinidae). Zool. Sci. 15: 425-431.

Komiyama T, Kobayashi H, Tateno Y, Inoko H, et al. (2009). An evolutionary origin and selection process of goldfish. Gene 430: 5-11.

Leclercq E, Taylor JF and Migaud H (2010). Morphological skin colour changes in teleosts. Fish. Fish. 11: 159-193.

Lou Y and Li XD (2006). Distant hybridization of fish and its application in aquaculture in China. J. Fish. Sci. Chin. 13: $151-158$.

Matsumoto J, Kajishima T and Hama T (1960). Relation between the pigmentation and pterin derivatives of chromatophores during development in the normal black and transparent scaled types of goldfish (Carassius auratus). Genetics 45 : 1177-1189.

Neumeyer C (2003). Wavelength dependence of visual acuity in goldfish. J. Comp. Physiol. A -Neuroethol. Sens. Neural Behav. Physiol. 189: 811-821.

Nilsson SH, Aspengren S and Wallin M (2013). Rapid color change in fish and amphibians - function, regulation, and emerging applications. Pigment Cell Melanoma Res. 26: 29-38.

Nirchio M, Fenocchio AS, Swarça AC, Pérez JE, et al. (2003). Cytogenetic characterization of hybrids offspring between Colossoma macropomum (Cuvier, 1818) and Piaractus brachypomus (Cuvier, 1817) from Caicara del Orinoco, Venezuela. Caryologia 56: 405-411.

Sugimoto M (2002). Morphological color changes in fish: regulation of pigment cell density and morphology. Microsc. Res. Tech. 58: 496-503.

Wang SY, Luo J, Murphy RW, Wu SY, et al. (2013). Origin of Chinese goldfish and sequential loss of genetic diversity accompanies new breeds. PLoS One. 8: e59571.

Wang ZH, Wang JS and Wang JL (2006). Culture and view of goldfish. Shanghai Scientific and Technical Press, $171-176$. Wu HS and Fu YY (1983). China goldfish. Tianjing Sci Press, 29-46.

Xu W (2009). Discussion on nomenclature and classification of the transparent goldfish. J. Shanghai Fish. Univ. 5: 559- 
564.

Xu W, Bai QL, Liu MH and Shen J (1999). Body color of filial generation from color crucian carp and red crucian carp. J. Fish. Sci. China 6: 33-36.

Xu W, Bai QL, Liu MH, Cao DC, et al. (2000). Studies on selective breeding and genetic characters of carnation crucian carp. Fish. Sci. China 4: 113-115.

Xu W, Cao DC and Li CT (2005). Preliminary observation on peritoneum's chromatophore of carp and crucian carp. Chin. J. Fish. 18: 66-68. 